\title{
ESTUDO DE TÉCNICAS DE CARACTERIZAÇÃO MICROESTRUTURAL DE AÇOS INOXIDÁVEIS DÚPLEX POR MEIO MICROSCOPIA ÓPTICA.*
}

Daniella Caluscio dos Santos ${ }^{1}$ Gustavo Lucacksak Barbosa ${ }^{27}$

\section{Resumo}

Objetivo desse trabalho consiste em avaliar a utilização de diferentes reagentes metalográficos na caracterização microestrutural das fases presentes nos aços inoxidáveis dúplex (AID) por estereologia quantitativa. Amostras do aço UNS S31803 foram solubilizadas e envelhecidas por 36h a 700, 850 e 950ㄷ. Foram estudados diferentes reagentes para a revelação de fases: Beraha modificado; $\mathrm{KOH}$; Murakami; e $\mathrm{NaOH}$. Após os ataques foi realizada quantificação de fases por estereologia quantitativa a partir de imagens de microscopia óptica (MO) que foram comparadas à quantificação realizada a partir de imagens de elétrons retroespalhados (MEV), e medidas magnéticas utilizando ferritoscópio. Para a condição solubilizada, os ataques $\mathrm{NaOH}$ e Beraha modificado são mais indicados. Para amostras envelhecidas a quantificação por MO mostra diferença significativa em relação à análise realizada por imagens de BSC. Particularmente para amostras envelhecidas a 700 e $850^{\circ} \mathrm{C}$, provavelmente relacionada à morfologia descontínua das fases intermetálicas encontradas nas nessas condições. Já para as amostras envelhecidas à $950^{\circ} \mathrm{C}$, onde a morfologia dessas fases é maciça em relação às demais temperaturas, esse comportamento é menos evidente.

Palavras-chave: Aço; Inoxidável; Duplex; Intermetálicas.

\section{STUDY OF MICROSTRUCTURAL CHARACTERIZATION TECHNIQUES OF DUPLEX STAINLESS STEELS BY OPTICAL MICROSCOPY}

\section{Abstract}

The aim of this work is evaluate the use of different metallographic etch in microsctructural characterization of phases found in duplex stainless steels (DSS) though quantitative stereology. Samples of UNS S31803 DSS were solution treated and aged for $36 \mathrm{~h}$ at 700,850 and $950^{\circ} \mathrm{C}$. Different metallographic etch were studied: Modified Beraha; $\mathrm{KOH}$; Murakami; and $\mathrm{NaOH}$. The etched samples were submitted to microstructural analysis using optical microscopy $(\mathrm{OM})$ and compared to the results obtained from quantitative analysis realized in backscattered electron images (BSE-MEV), and magnetic measurements using a ferritscope. For solution treated samples, $\mathrm{NaOH}$ and Modified Beraha were considered the most suitable etch. For aged samples the quanfification through $\mathrm{OM}$ shows a significant difference in relation to $\mathrm{BSC}$ in all the aged sample. In particular at 700 and $850^{\circ} \mathrm{C}$ the reported differences are probably related to discontinuous morphology of intermetallic phases found in this conditions. For the samples aged at $950^{\circ} \mathrm{C}$, where the morphology of this phases is massive in relation to the other temperatures, this behavior is more evident.

Keywords: Steel; Stainless; Duplex; Intermetallic. 
1 Engenheira Química, Doutora, Professora Adjunta, Departamento de Engenharia de Materiais Centro Universitário da FEl, São Bernardo do Campo, São Paulo, Brasil.

2 Estudante de Engenharia de Materiais, Estudante, Departamento de Engenharia de Materiais Centro Universitário da FEl, São Bernardo do Campo, São Paulo, Brasil. 


\section{INTRODUÇÃO}

Os aços inoxidáveis são considerados dúplex quando possuem frações volumétricas aproximadamente iguais de austenita $(\gamma)$ e ferrita $(\alpha)$ e apresentam não só excelentes propriedades mecânicas, mas também à corrosão. Isso faz com que esses materiais sejam utilizados principalmente nas indústrias química, petroquímica, offshore e papel e celulose. Entretanto, quando submetidos a altas temperaturas os aços inoxidáveis dúplex podem formar fases intermetálicas, como sigma $(\sigma)$ e chi $(\chi)$, ricas em $\mathrm{Cr}$ e Mo, que podem afetar tanto a tenacidade quanto a resistência à corrosão desses materiais [1-3].

Existem diversos meios para a identificação e quantificação de fases intermetálicas, dentre elas: microscopia óptica (MO), microscopia eletrônica de varredura (MEV), análises magnéticas e difratometria de raios $X$ (DRX). Todavia, existem limitações em todas as técnicas citadas, particularmente para a quantificação dessas fases que podem ser encontradas em frações muito baixas no início de sua formação.

Por MEV é possível identificar fases intermetálicas como sigma e chi numa matriz composta por ferrita e austenita. Utilizando imagens de elétrons retroespalhados (BSC-Backscattered Electron Image) é possível identificar fases intermetálicas pois por serem ricas em elementos com peso atômico elevado, como $\mathrm{Cr}$ e $\mathrm{Mo}$, se apresentam com coloração mais clara [4]. A diferença de composição química entre sigma e chi, que contém mais Mo, faz com que sua identificação e quantificação seja possível por meio dessa técnica como alguns trabalhos desse grupo de pesquisa reportaram em outras publicações [5-7]. Uma limitação desta técnica está na diferenciação entre ferrita e austenita por meio de BSC, pois as diferenças de composição química entre as fases, não são suficientes para gerar contraste necessário para a análise quantitativa por meio de software de análise de imagens. Para esse fim, normalmente num aço dúplex a medição das frações de ferrita e austenita são feitas por ferritoscópio ou difração de raios $X$.

Embora a análise por MEV seja uma ferramenta usual para se identificar e, a partir de suas imagens, quantificar as fases intermetálicas presentes no material, há a necessidade da exploração de formas mais simples e rápidas para realizar o mesmo processo. Para tal, a identificação de fases é possível utilizando microscopia óptica (MO) onde se faz necessário uso de reagentes adequados para caracterizar as fases intermetálicas. A grande dificuldade da utilização de MO para identificação e quantificação de fases intermetálicas nos aços inoxidáveis dúplex consiste na escolha do reagente metalográfico mais adequado, pois esses materiais apresentam excelente resistência à corrosão [8]. Por meio de revisão minuciosa da literatura, os reagentes mais utilizados para a caracterização microestrutural de aços dúplex são: Beraha modificado; $\mathrm{KOH}$; Murakami; Villela; $\mathrm{NaOH}$ e Aqua Régia, cujas composições químicas são apresentadas na Tabela 1.

Tabela 1. Reagentes metalográficos utilizados no trabalho.

\begin{tabular}{|ccccc|}
\hline Ataque & Natureza & Composição química da solução & Ref. \\
\hline Murakami & Imersão a $80^{\circ} \mathrm{C}$ & $10 \mathrm{~g} \mathrm{~K}{ }_{3} \mathrm{Fe}(\mathrm{CN})_{6} ; 10 \mathrm{~g} \mathrm{KOH} 100 \mathrm{ml} \mathrm{H}_{2} \mathrm{O}$ destilada & $(9)$ \\
\hline $\mathrm{KOH}$ & Eletrolítico $5 \mathrm{VcC}$ & $10 \% \mathrm{KOH}$ & $(10)$ \\
\hline $\begin{array}{c}\text { Beraha } \\
\text { Modificado }\end{array}$ & Imersão & $2 \mathrm{~g}\left(\mathrm{NH}_{4} \mathrm{HF}_{2}\right) ;$ para $100 \mathrm{ml} \mathrm{H}_{2} \mathrm{O}$ destilada & $(9)$ \\
\hline $\mathrm{NaOH}$ & Eletrolítico $3 \mathrm{Vcc}$ & $10 \mathrm{M} \mathrm{NaOH}$ & $(11)$ \\
\hline
\end{tabular}


Diante do exposto, o objetivo desse trabalho é avaliar a utilização de diferentes reagentes metalográficos na caracterização microestrutural quantitativa das fases presentes nos aços inoxidáveis dúplex por meio de software de análise de imagens. Os resultados obtidos por essa técnica serão comparados aos encontrados por imagens de BSC.

\section{MATERIAIS E MÉTODOS}

O material em estudo foi adquirido como chapa de $3 \mathrm{~mm}$ de espessura laminada a $1100^{\circ} \mathrm{C}$ e resfriada por jato de ar e água forçado. A composição química do material é apresentada na Tabela 2.

Tabela 2 - Composição do aço UNS S31803 (\% em massa)

\begin{tabular}{lllllllllllll}
\hline $\mathrm{Cr}$ & $\mathrm{Ni}$ & $\mathrm{Mo}$ & $\mathrm{Mn}$ & $\mathrm{N}$ & $\mathrm{C}$ & $\mathrm{Si}$ & $\mathrm{Cu}$ & $\mathrm{Co}$ & $\mathrm{P}$ & $\mathrm{S}$ & $\mathrm{Nb}$ & $\mathrm{Fe}$ \\
\hline 22,48 & 5,74 & 3,20 & 1,42 & 0,162 & 0,018 & 0,35 & 0,15 & 0,07 & 0,019 & 0,001 & 0,006 & Bal. \\
\hline
\end{tabular}

Todas as amostras foram submetidas a um tratamento térmico de solubilização a $1090^{\circ} \mathrm{C}$ por 30 min para que a estrutura de partida fosse composta apenas por ferrita e austenita em frações aproximadamente iguais. A temperatura de solubilização foi prevista por simulação de equilíbrio realizada com auxílio do software ThermoCalc@5 e com auxílio da base de dados TCFE8, apresentada na Figura 1.

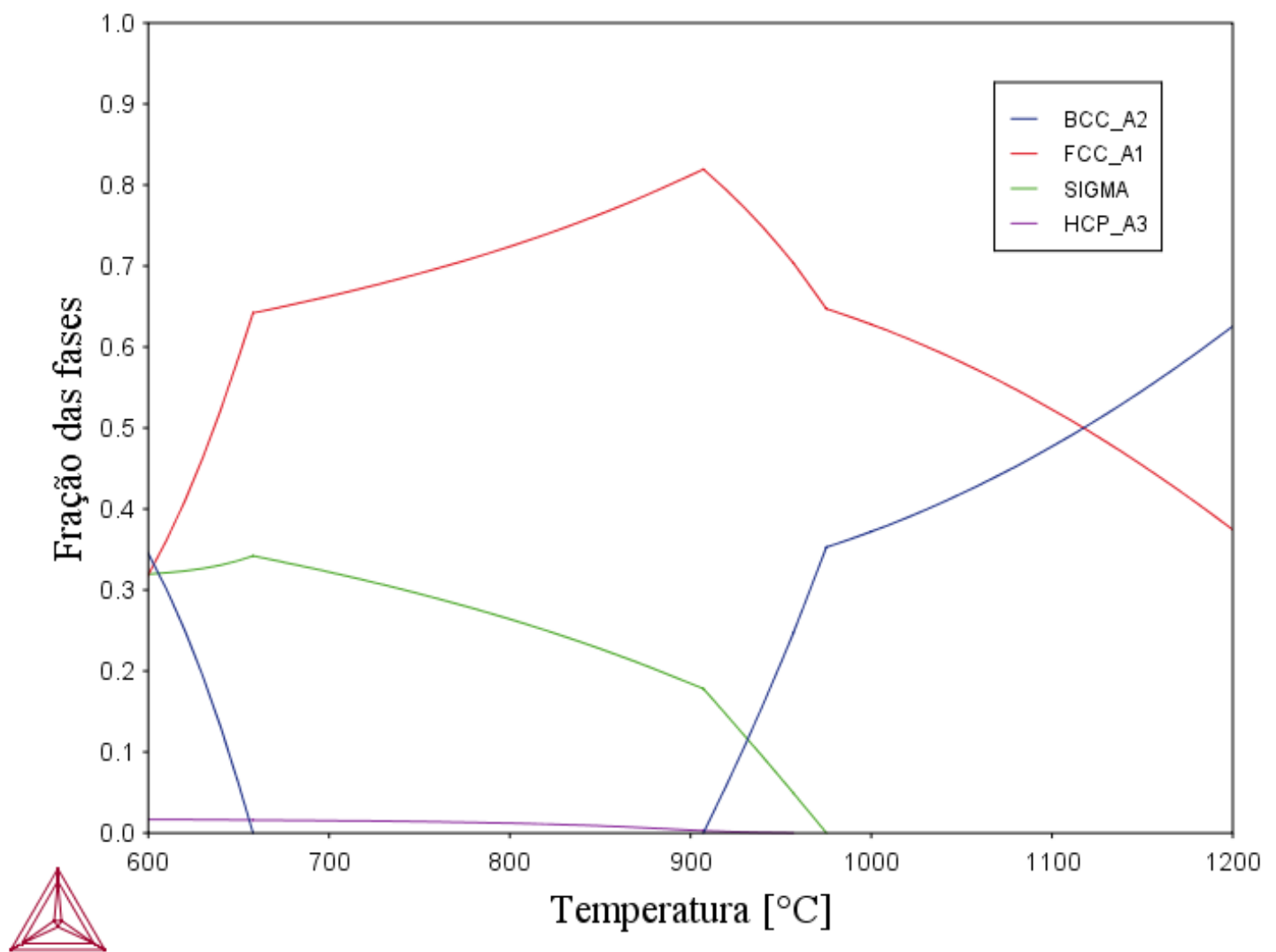

Figura 1. Frações das fases em função da temperatura para o aço UNS S31803 de composição indicada na Tabela 2 utilizando software ThermoCalc@5 na base de dados TFCE8: Ferrita (BCC_A2); Austenita (FCC_A1); Sigma (SIGMA); Nitretos de Cromo (HCP_A3). 
As amostras solubilizadas foram envelhecidas por $36 \mathrm{~h}$ a 700,850 e $950^{\circ} \mathrm{C}$ visando a formação de fases intermetálicas com diferentes morfologias, com resfriamento em água. Em seguida as amostras foram cortadas, dando origem a corpos de prova com superfície de observação correspondente à seção longitudinal das chapas, lixadas e polidas até acabamento superficial de $1 \mu \mathrm{m}$. As frações volumétricas das fases foram obtidas por meio de metalografia quantitativa após ataques por MO utilizando os ataques citados na Tabela 1. Essas frações foram quantificadas utilizando do Software Olympus AnalySIS Docu parte integrante de microscópio Olympus em 30 campos por amostra (Figura 2).

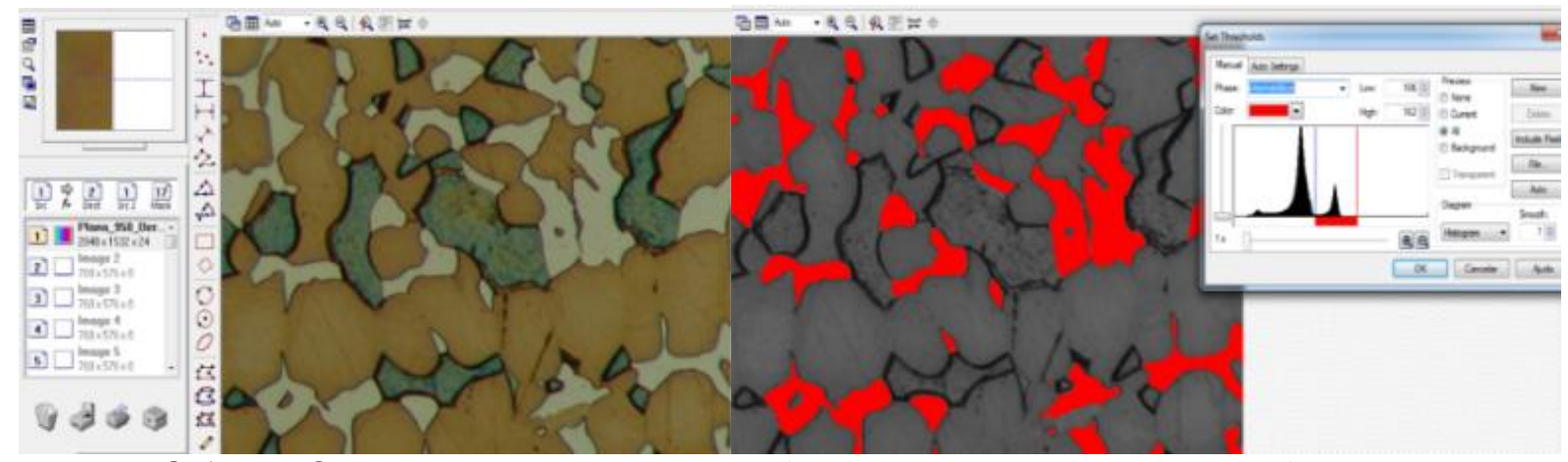

Figura 2. Software Olympus Analysis Docu onde são ajustados os limites de detecção de cada uma das imagens. Amostra envelhecida por $36 \mathrm{~h}$ a $950^{\circ} \mathrm{C}$.

Para comparação, as frações de fases foram determinadas utilizando imagens BSC obtidas por MEV, analisadas no mesmo software de análise de imagens, utilizando 10 campos por amostra. A caracterização por MEV foi realizada em microscópio eletrônico CAMSCAN CS3200LV do CDMatM-FEI. A fração de ferrita foi comparada ainda ao método tradicional por medidas magnéticas com o auxílio de um ferritoscópio FISCHER modelo MP30 com limite de detecção de 0,1\%. Com auxílio da medida magnética da porcentagem de ferrita foi possível quantificar a porcentagem de austenita presente no material a partir da equação (1). Para a realização dos ataques eletrolíticos, foi necessária a utilização de equipamento para ataque eletrolítico Struers LectroPol-5 e manta térmica para atingir temperaturas requeridas nos ataques à quente.

$$
\% \gamma=100-\% \alpha-\%(\sigma+\chi)
$$

\section{RESULTADOS E DISCUSSÃO}

As micrografias obtidas por BSC (MEV) na amostra solubilizada mostram a estrutura de ferrita e austenita e a ausência de fases intermetálicas (Figura 2.a) como previsto anteriormente pelo ThermoCalc(c). Os ataques químicos realizados nessa amostra (Figura 3) utilizando diferentes reagentes, mostram contraste entre a ferrita (escura) e austenita (clara). Entretanto, somente nos ataques de Beraha e $\mathrm{NaOH}$ (Figuras 3.c e e) é possível realizar a quantificação automática de ferrita que resultou respectivamente numa porcentagem em volume de em $56,8 \pm 3,3 \%$ e $57,0 \pm 2,6 \%$. Na amostra atacada com reagente de $\mathrm{KOH}$ houve corrosão na interface ferrita/austenita. Dessa maneira, o ataque permite a identificação das fases, mas impede o ajuste da tonalidade necessária para quantificação de fases utilizando software de análise de imagens. 
O resultado de ambos os ataques são bastante próximos considerando o desvio padrão das medidas e superiores aos encontrados por ferritoscópio $(52,9 \pm 2,5 \%)$ cujas medidas podem ter sido influenciadas pelo bandeamento característico da estrutura duplex estudada, como reportado anteriormente na literatura[12]. Os resultados de quantificação obtidos são apresentados na Figura 4.

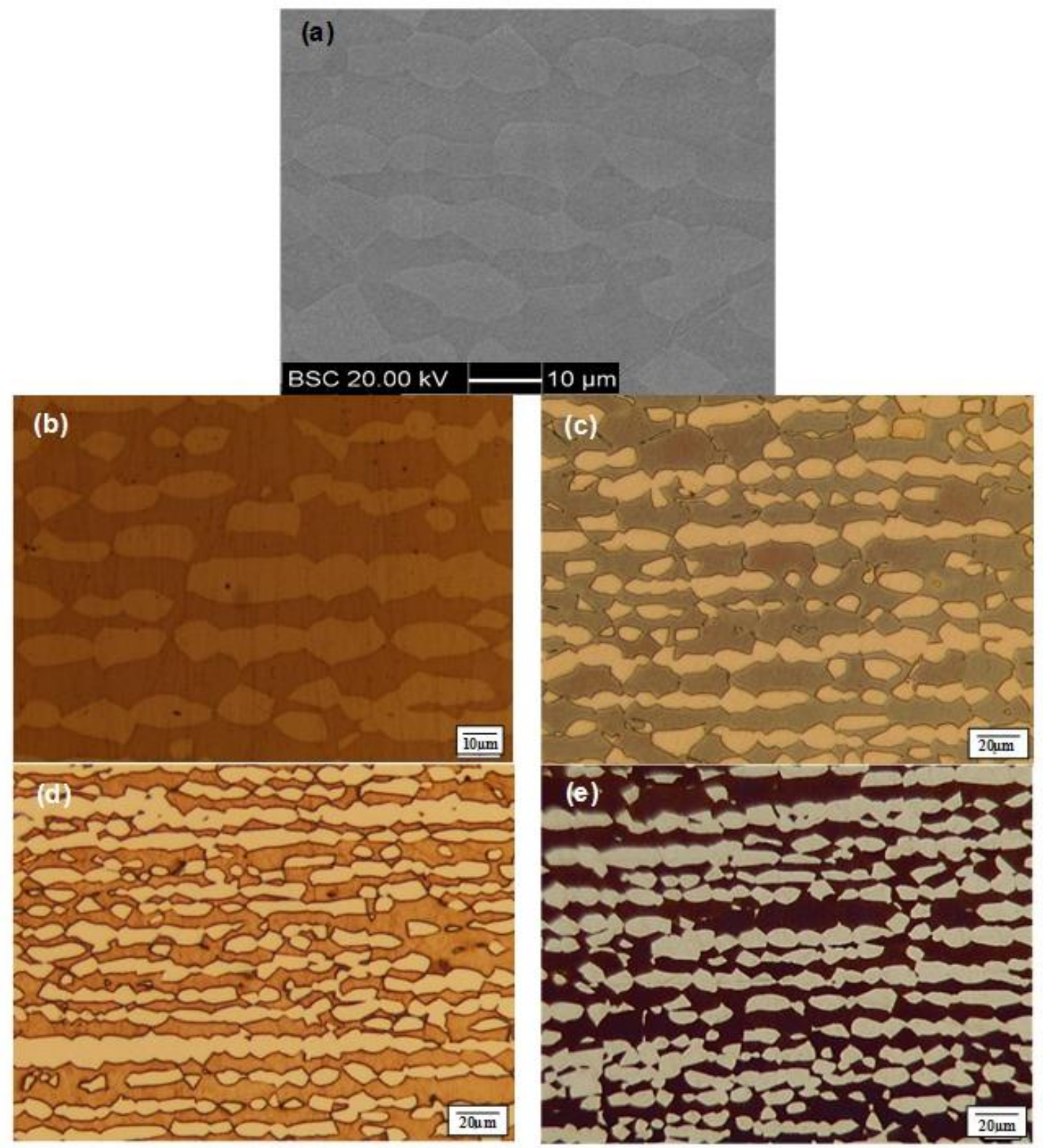

Figura 3. Aço UNS S31803 solubilizado: (a) Imagem de elétrons retroespalhados (BSC) sem ataque; (b) MO Murakami,4min a $80^{\circ} \mathrm{C}$; (c) $\mathrm{MO} \mathrm{NaOH}, 3$ Vcc por $10 \mathrm{~s}$; (d) $\mathrm{MO} \mathrm{KOH}, 5 \mathrm{Vcc}$ por 45s; (e) MO Beraha Modificado (até 1min). 


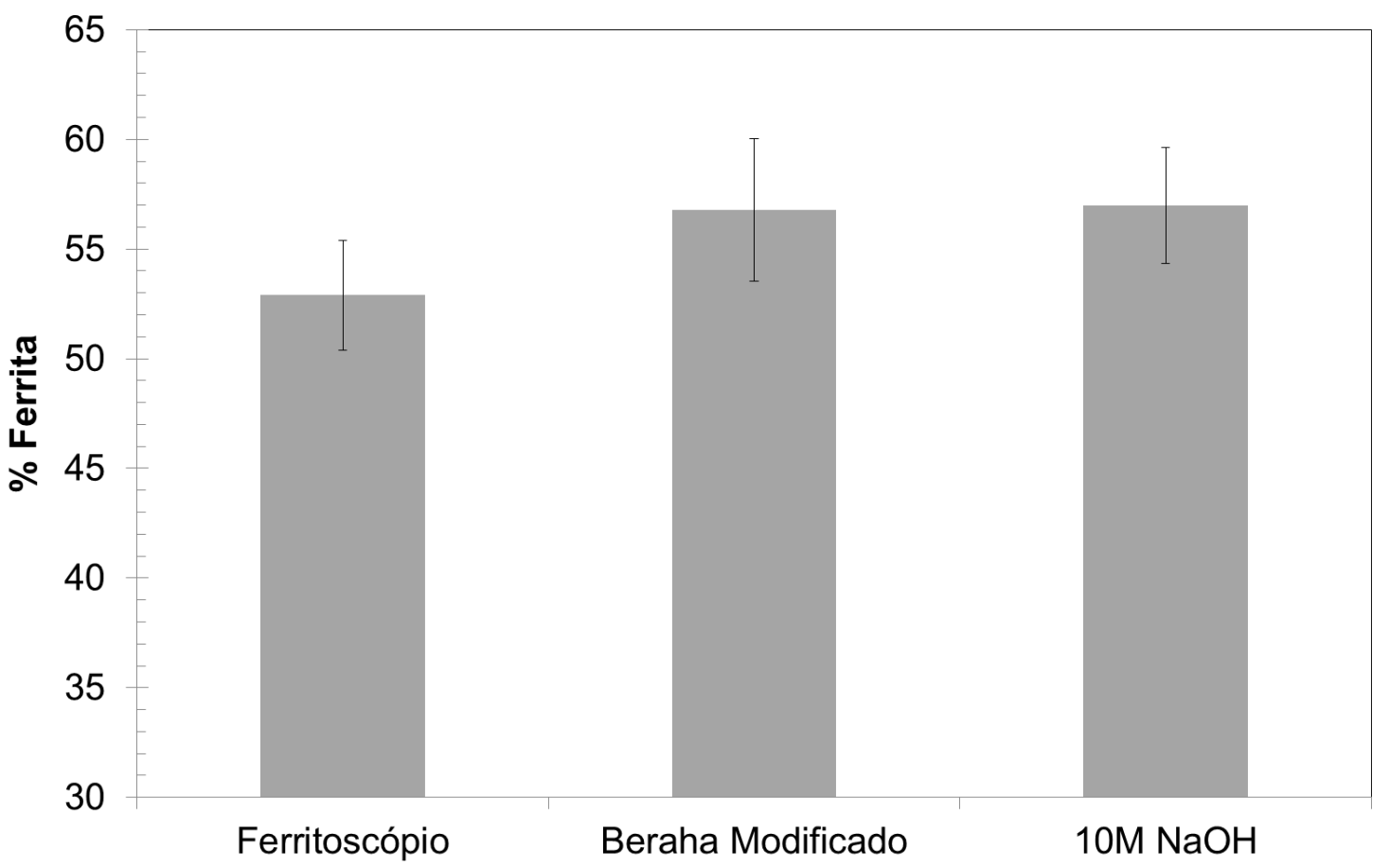

Figura 4. Gráfico comparativo utilizando diferentes técnicas para quantificação da fração de ferrita da amostra solubilizada.

Tratando-se da quantificação de fases utilizando a amostra solubilizada, o ataque de Beraha modificado e o ataque de $\mathrm{NaOH}$ trazem valores muito próximos de fração de ferrita. Entretanto, o ataque de Beraha possui grande importância quando se trata de amostras envelhecidas, como será discutido na sequência.

Nas amostras envelhecidas (Figura 5) é possível se observar a formação de fases intermetálicas nas imagens de elétrons retroespalhados, sigma (cinza clara) e chi (branca) formadas preferencialmente nas interfaces ferrita/austenita, com posterior crescimento em direção à ferrita, que é fonte de $\mathrm{Cr}$ e Mo para a formação dessas fases. É possível se observar a provável formação de chi na amostra envelhecida a $700^{\circ} \mathrm{C}$ (Figura 5.a), que é uma fase metaestável e não aparece na simulação de equilíbrio mostrada na Figura 1. Acima dessa temperatura, chi pode ter se transformado em sigma, como indicam algumas referências da literatura [1-4]. Foi realizada a análise por espectroscopia por energia dispersiva (EDS) nas amostras envelhecidas a $700^{\circ} \mathrm{C}$ a fim de identificar as fases intermetálicas formadas. Essa análise é apresentada na Figura 6. Pode-se observar que a fase mais clara mostrada na imagem de elétrons retroespalhados é mais rica em Mo, como comentado anteriormente.

Observa-se ainda que a medida que a temperatura de envelhecimento se eleva, a microestrutura de sigma evolui de descontínua a $700^{\circ} \mathrm{C}$ para maciça a $950^{\circ} \mathrm{C}$, provavelmente relacionada à baixa taxa de nucleação e alta taxa de difusão em temperaturas superiores. 


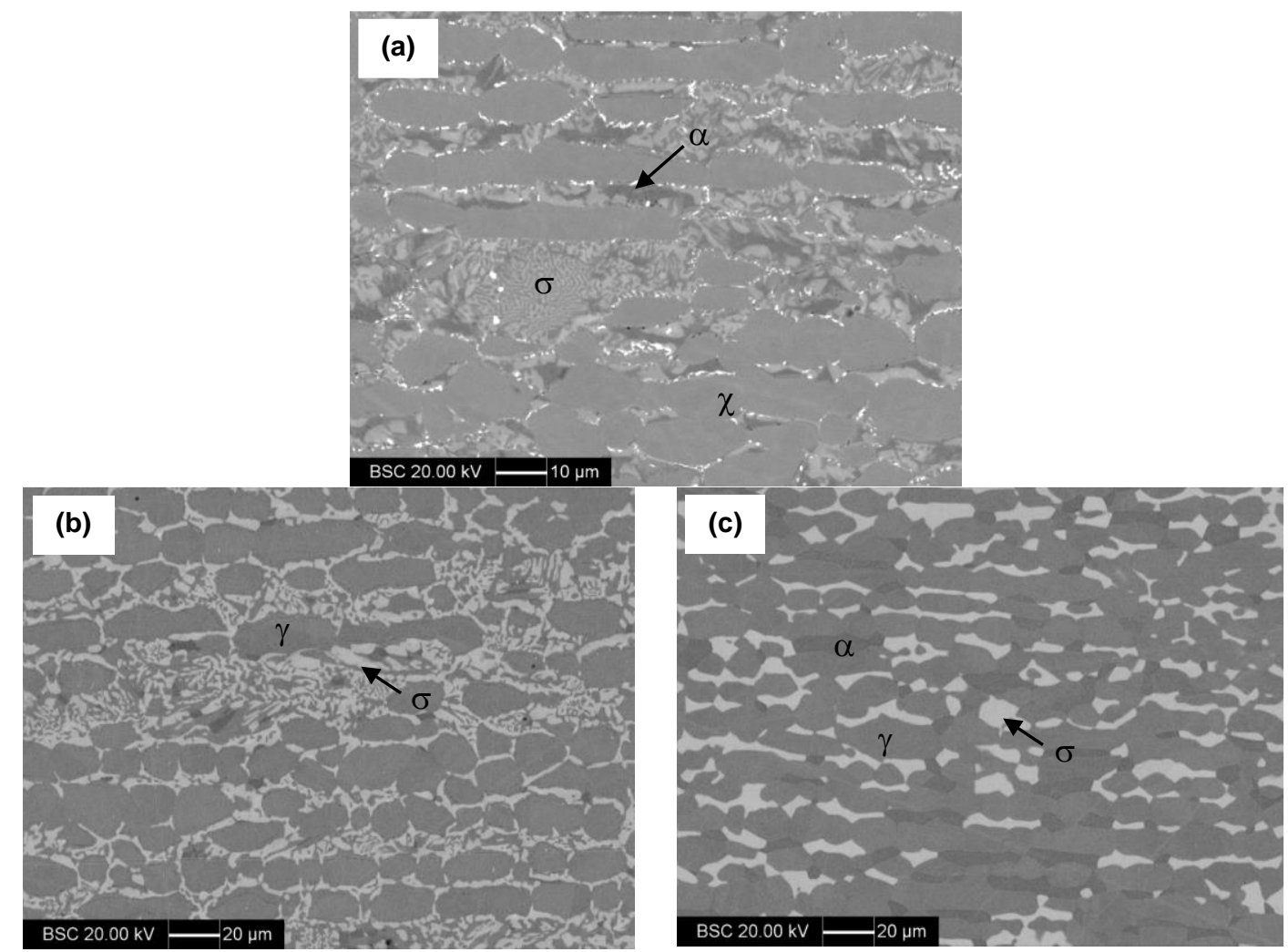

Figura 5. Aço UNS S31803 envelhecido por 36 a: (a) $700^{\circ} \mathrm{C}$, (b) $850^{\circ} \mathrm{C}$ e (c) $950^{\circ} \mathrm{C}$. Imagem de elétrons retroespalhados (BSC) sem ataque.

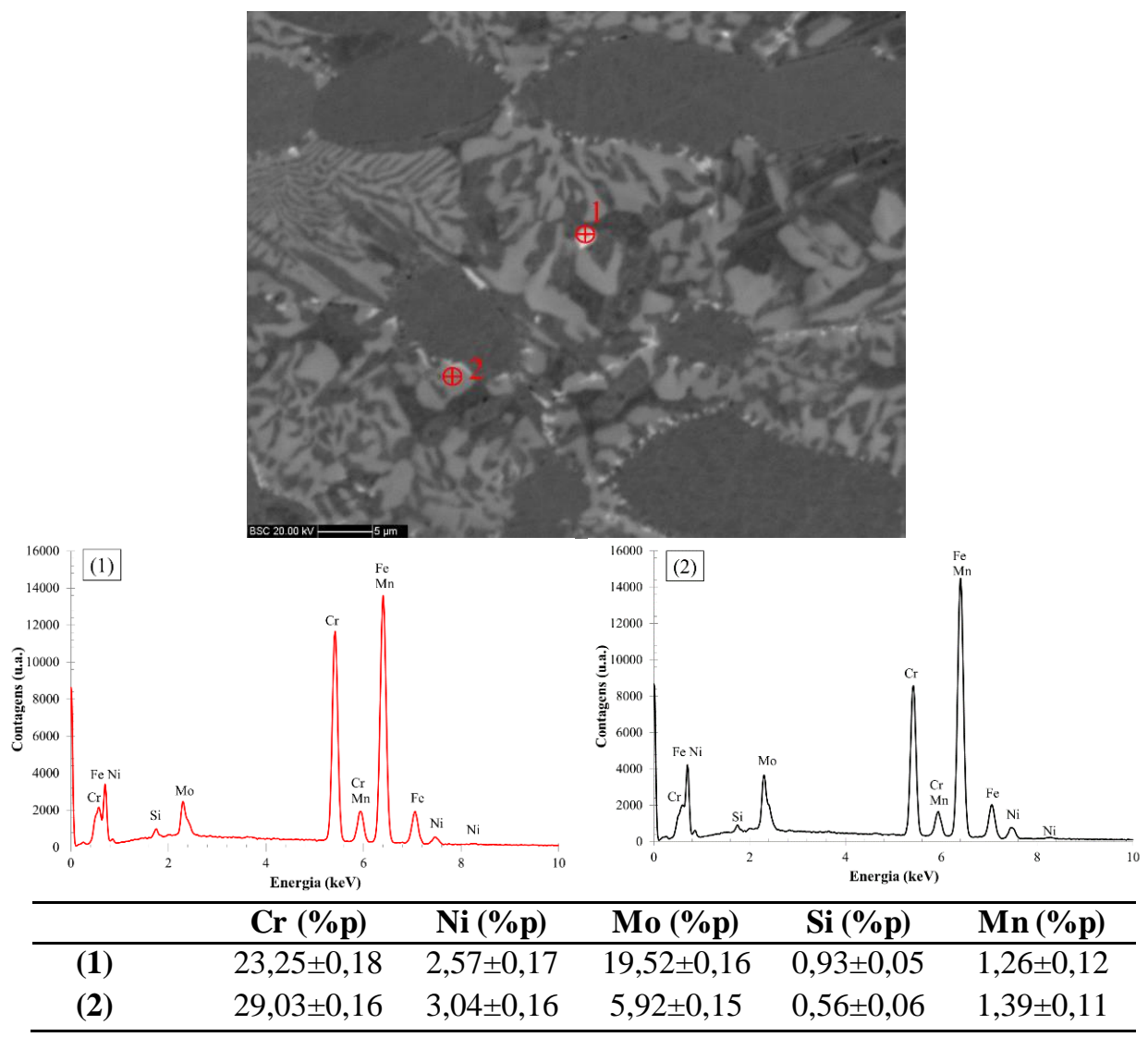

Figura 6. Análise por EDS da amostra envelhecida por $36 \mathrm{~h}$ a $700^{\circ} \mathrm{C}$ 
Analisando as mesmas amostras analisadas por MEV, agora atacadas com os regentes metalográficos citados na Tabela 1, foram obtidas as micrografias apresentadas na Figura 7. Nessa figura se observa que os ataques de (a) Murakami, (b) $\mathrm{KOH}$ e (d) $\mathrm{NaOH}$ revelam apenas as fases intermetálicas permanecendo a matriz sem ataque. O ataque de Beraha modificado (Figura 7.c), é o único dos estudados que distingue as fases da matriz (ferrita e austenita) das fases intermetálicas formadas. Entretanto, as fases sigma e chi não podem ser distinguidas por meio desses ataques. A quantificação de fases intermetálicas feita para as amostras envelhecidas segue apresentada na Figura 8 que mostra suas respectivas frações em função do tipo de ataque realizado e sua comparação aos dados obtidos por imagens de BSC. De maneira geral é possível perceber que dentre as amostras apresentadas, na amostra envelhecida a $700^{\circ} \mathrm{C}$ é a que apresenta morfologia descontínua e mostra maior diferença em relação às medidas das imagens BSC. Esse comportamento se justifica, pois, o ajuste do delineamento das fases é mais difícil para essas amostras em comparação às que apresentam morfologia mais maciça, como na amostra envelhecida a $950^{\circ} \mathrm{C}$.

Além disso, à exceção do ataque de Beraha modificado, os demais ataques levam à dissolução das fases intermetálicas que resultam na formação de relevo entre essa fase a matriz. Esse comportamento dificulta o ajuste de foco do microscópio óptico, que pode levar à desvios na quantificação das fases. Dessa maneira, quanto maior o número de interfaces entre as fases, maior os desvios encontrados nas quantificações.

Em contrapartida, nas amostras envelhecidas a $950^{\circ} \mathrm{C}$ a morfologia de sigma maciça, contribui para o ajuste do delineamento das fases, e consequentemente para sua quantificação, trazendo resultados mais próximos aos obtidos a partir de estereologia quantitativa utilizando imagens de BSC.

\section{CONCLUSÃo}

Esse trabalho revela que a quantificação de fases a partir do reagente de Beraha modificado e o ataque eletrolítico de $\mathrm{NaOH}$ são os mais indicados para amostras solubilizadas pois diferenciam adequadamente as fases ferrita e austenita e se aproximam às medidas realizadas por ferritoscópio.

Para amostras envelhecidas a quantificação por meio de análise metalográfica por microscopia óptica mostra uma diferença significativa em relação à análise realizada por imagens de elétrons retroespalhados para amostras envelhecidas a 750 e $850^{\circ} \mathrm{C}$. Esse comportamento é provavelmente relacionado à morfologia descontínua das fases intermetálicas encontradas nas amostras, o que dificulta a seleção e a quantificação dessas regiões por meio de software de análise de imagens. Já para a temperatura de $950^{\circ} \mathrm{C}$, onde a morfologia dessas fases é maciça em relação às anteriores esse comportamento é menos evidente.

Por meio dos ataques metalográficos testados é impossível diferenciar as fases sigma e chi, sendo essa possível somente por meio das imagens de MEV. 
$700^{\circ} \mathrm{C}$

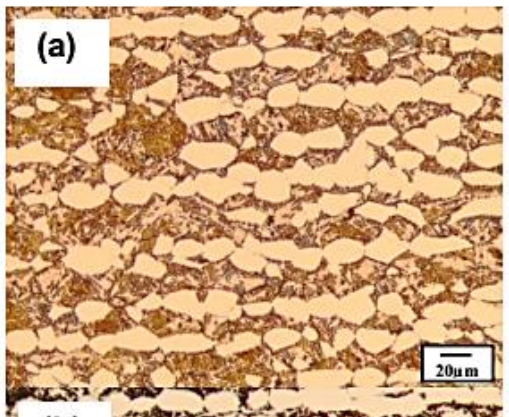

(b)

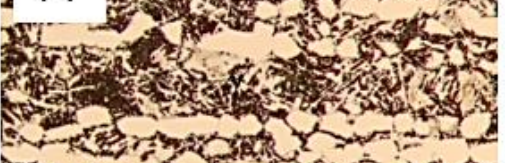
-

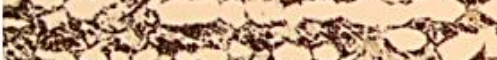

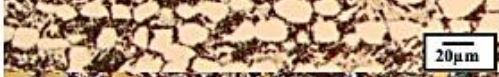
(c)

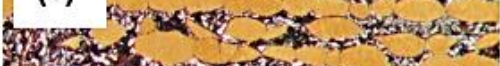

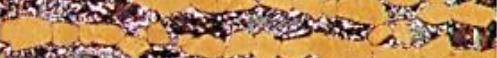
A

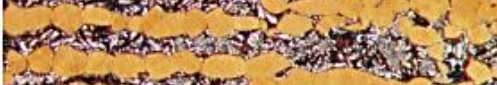
2.

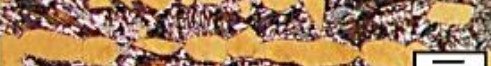

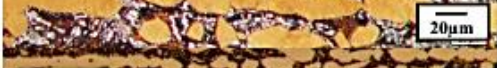

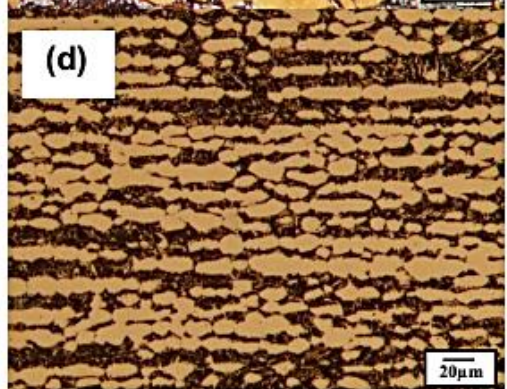

$850^{\circ} \mathrm{C}$
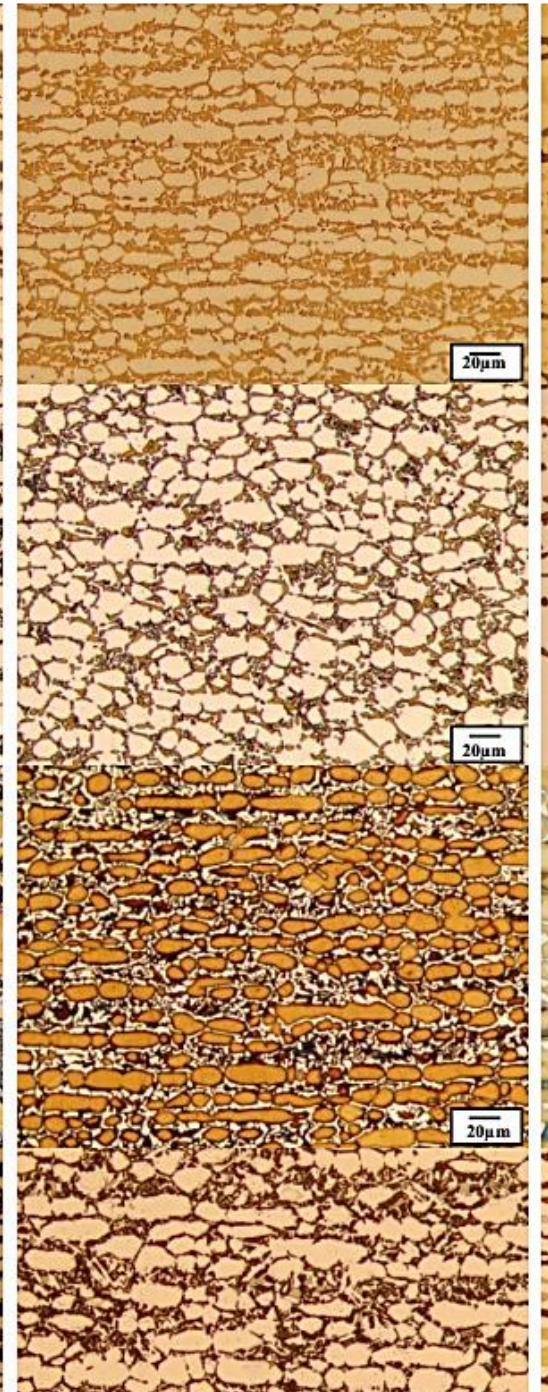

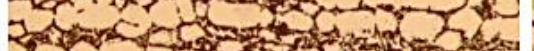
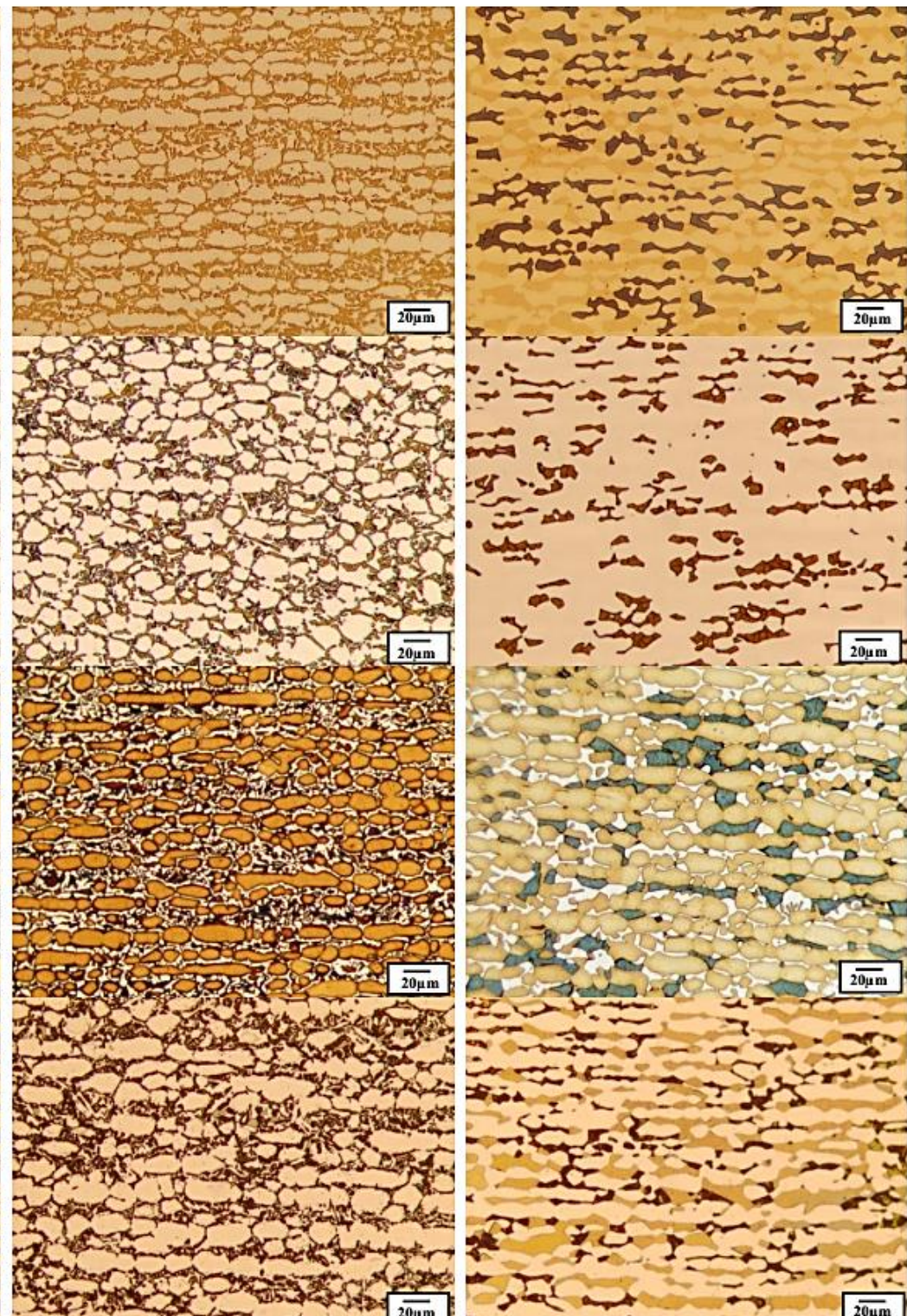

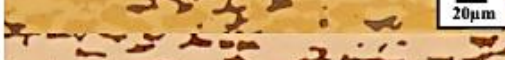

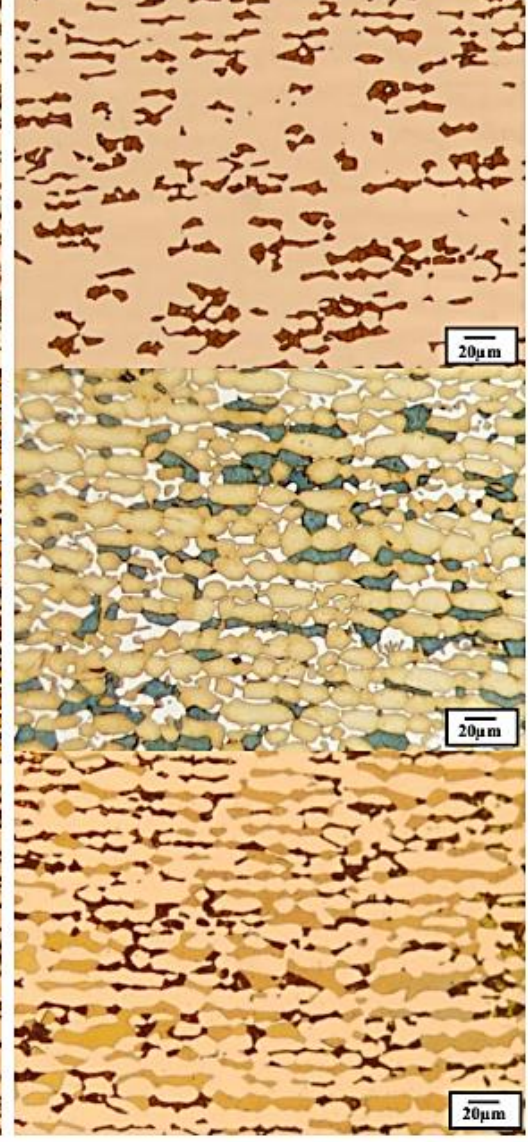

Figura 7. Imagens de microscopia óptica de amostras do aço UNS $\$ 31803$ envelhecido a 750, 850 e $950^{\circ} \mathrm{C}$ : (a) Murakami, $4 \mathrm{~min}$ a $80^{\circ} \mathrm{C}$; (b) $\mathrm{KOH}, 5 \mathrm{Vcc}$ por $45 \mathrm{~s}$; (c) Beraha Modificado (até $1 \mathrm{~min}$ ); (d) $\mathrm{NaOH}, 3 \mathrm{Vcc}$ por $10 \mathrm{~s}$. 


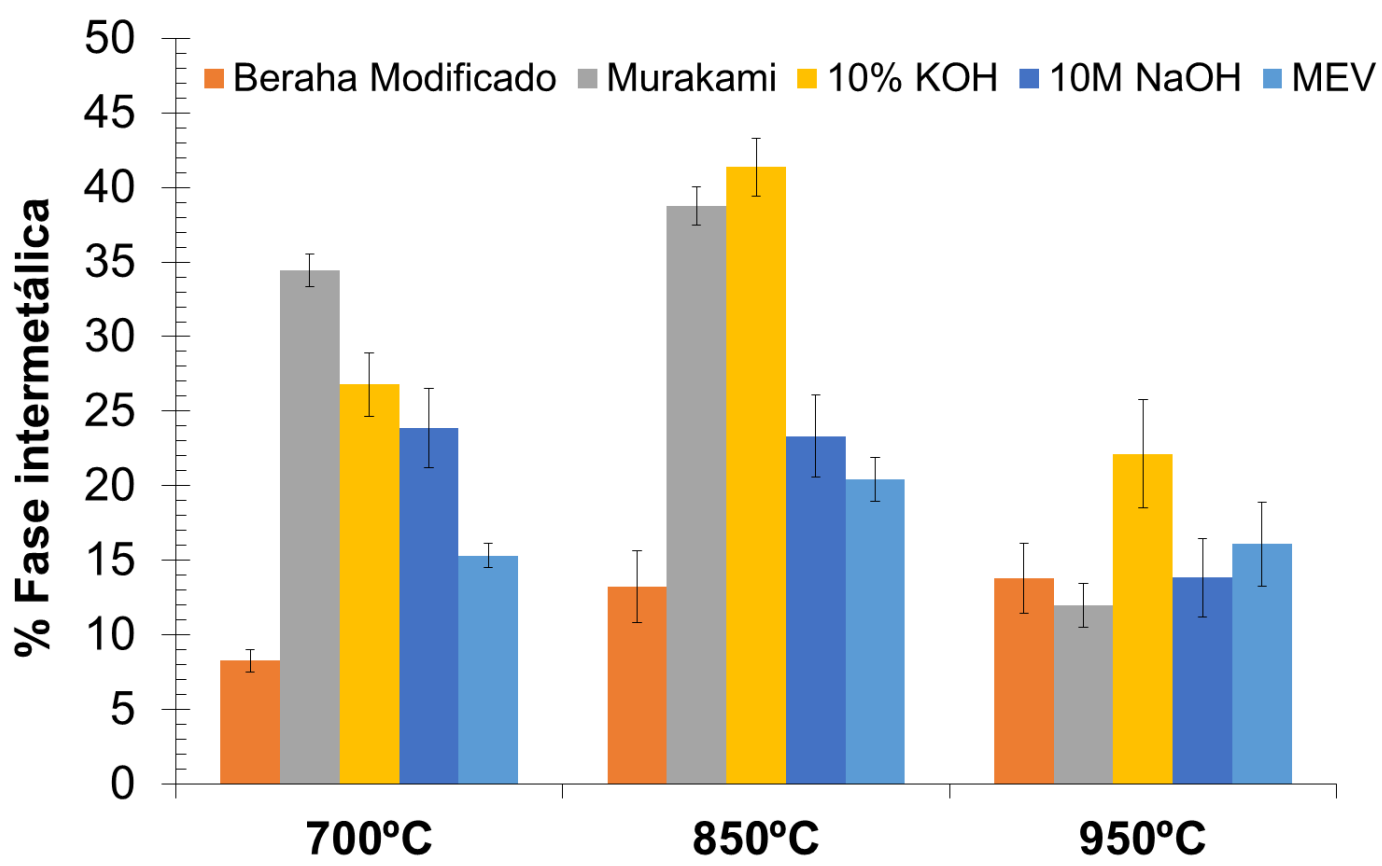

Figura 8. Gráfico comparativo para os diferentes ataques metalográficos em função da \% de fase intermetálica (sigma+chi) na direção longitudinal.

\section{REFERÊNCIAS}

1 Charles J. Dúplex stainless steels, a review after DSS'07 held in Grado. La Revue de Métallurgie. 2008; 155-171.

2 Nilsson, JO. Super duplex stainless steel. Materials Science and Technology. 1992;8: 685-700.

3 Solomon HD; Devine, T.M. Jr. Duplex stainless steel: a tale of two phases. In: International On Conference Duplex Stainless Steels, Proceedings; 1982; St Louis, Metals Park, Ohio: ASM, 1982. p. 693-756.

4 Escriba DM, Materna-Morris E, Plaut RL, Padilha AF. Chi-phase precipitation in a duplex stainless steel. Materials Characterization, 2009; 60(11): 1214-1219.

5 Santos DC; Magnabosco R. Kinetic Study to Predict Sigma Phase Formation in Duplex Stainless Steels. Metallurgical and Materials Transactions A, 2016; 47(4): 1554-1565.

6 Santos DC, Magnabosco R, Moura Neto C. Influence of Sigma Phase Formation on Pitting Corrosion of an Aged UNS S31803 Duplex Stainless Steel. 2013; 69(9): 900-911.

7 Santos DC, Magnabosco R, Moura Neto C. Estudo dos mecanismos e da cinética de formação de fase sigma no aço inoxidável dúplex UNS S31803 envelhecido a $700^{\circ} \mathrm{C}$. In: Associação Brasileira de Metalurgia, Materiais e Mineração. Anais do $68^{\circ}$ Congresso da ABM Internacional; 2013; Belo Horizonte, Brazil. São Paulo:ABM;2013.p .68-79.

8 Bramfitt BL Benscoter AO. Metallographer's Guide. Ohio: ASM International; 2002, 354p.

9 Van der Voort GF, Lucas GM. Metallography and Microstructures of Stainless Steels and Maraging Steels. In: ASM. Metallography and Microstructures. Ohio: ASM Handbook; 9, 2004. 
10 Elmer JW, Palmer TA, Specht ED. Direct Observations of Sigma Phase Formation in Duplex Stainless Steels Using In -Situ Synchrotron X-Ray Diffraction. Materials Science and Engineering. 2007; 9: 464-475.

11 Chen, TH, Yang, JR. Effects of solution treatment and continuous cooling on $\sigma$ phase precipitation in a 2205 duplex stainless steel. Materials Science and Engineering. 2001; 311: 28-41.

12 Magnabosco,R; Spomberg,S. Comparative study of ferrite quantification methods applied to duplex stainless steels. In: 7th European Stainless Steel Conference - Science and Market. Proceedings...; 2011; Como, Italia. Milão: AIM, 2011. 